\title{
Serum Osteocalcin Is Associated with Inflammatory Factors in Metabolic Syndrome: A Population-Based Study in Chinese Males
}

\author{
Ming Liao, , 2,3,4,5 Lirong Huang, ${ }^{2,3,4}$ Yan Mao, ${ }^{6}$ Yonghua Jiang, ${ }^{2,3,4}$ \\ Ziting Yao, ${ }^{2,3,4}$ Xinggu Lin, ${ }^{2,3,4}$ Zheng Lu, ${ }^{1,2}$ Chunlei Wu, ${ }^{2,7}$ Xue Qin, ${ }^{2,8}$ \\ Haiying Zhang, ${ }^{2,9,10}$ and Zengnan Mo $\mathrm{M}^{1,2,3,4}$ \\ ${ }^{1}$ Institute of Urology and Nephrology, First Affiliated Hospital of Guangxi Medical University, Nanning, Guangxi 530021, China \\ ${ }^{2}$ Center for Genomic and Personalized Medicine, Guangxi Medical University, Nanning, Guangxi 530021, China \\ ${ }^{3}$ Guangxi Key Laboratory of Genomic and Personalized Medicine, Nanning, Guangxi 530021, China \\ ${ }^{4}$ Guangxi Collaborative Innovation Center for Genomic and Personalized Medicine, Nanning, Guangxi 530021, China \\ ${ }^{5}$ Department of Biostatistics and Epidemiology, Perelman School of Medicine, University of Pennsylvania, \\ Philadelphia, PA 19014, USA \\ ${ }^{6}$ Department of Oncology, Third Affiliated Hospital of Guangxi Medical University, Nanning, Guangxi 530031, China \\ ${ }^{7}$ Department of Urology, First Affiliated Hospital of Xinxiang Medical College, Xinxiang, Henan 453100, China \\ ${ }^{8}$ Department of Clinical Laboratory, First Affiliated Hospital of Guangxi Medical University, Nanning, Guangxi 530021, China \\ ${ }^{9}$ School of Public Health, Guangxi Medical University, Nanning, Guangxi 530021, China \\ ${ }^{10}$ General Practice School, Guangxi Medical University, Nanning, Guangxi 530021, China
}

Correspondence should be addressed to Zengnan Mo; zengnanmo@hotmail.com

Received 13 February 2015; Revised 9 July 2015; Accepted 12 July 2015

Academic Editor: Fumio Tsuji

Copyright ( $) 2015$ Ming Liao et al. This is an open access article distributed under the Creative Commons Attribution License, which permits unrestricted use, distribution, and reproduction in any medium, provided the original work is properly cited.

Osteocalcin $(\mathrm{OCN})$ was potentially associated with inflammatory factors, so we explored the metabolic role in this association in general population. Our findings suggest that $\mathrm{OCN}$ was positively associated with IgG while inversely associated with $\mathrm{C} 3$, both of which were probably mediated by obesity. Moreover, serum OCN was inversely associated with hsCRP in men with impaired fasting glucose, hyperglycemia, or metabolic syndrome, while its association with IgE was significantly observed in men with a normal metabolic profile.

\section{Introduction}

Osteocalcin $(\mathrm{OCN})$ is an osteoblast-derived protein acting as a hormone stimulating insulin sensitivity, insulin secretion, and energy expenditure [1]; however, its effect on immune response and inflammatory cells has not been described before [2-4]. We previously reported that low level of serum total OCN was a potential marker of metabolic syndrome (MetS) that is characteristic of persistent low-grade systemic inflammation [5]. High sensitive C-reaction protein (hsCRP), as a sensitive physiological marker of subclinical systemic inflammation, has been shown to contribute to individual components of MetS [6], but its association with OCN was not clearly studied. In a population-based study assessing the risk of nontraumatic fractures, serum total OCN as a marker of bone turnover showed an inverse relation to hsCRP, without consideration of the metabolic confounders [7]. In studies assessing metabolic factors, the association between serum total OCN and hsCRP appeared to be easily observed in postmenopausal women [8] or young overweight and obese women [9]; however, the other studies showed that the association was independent of sex, in type 2 diabetic patients [10] or old adults (aged $\geq 65$ years) [11]. In one study enrolling solely adult males, serum total OCN was inversely associated with CRP in those with risk factors for cardiovascular diseases, but the significant association 
disappeared after adjusting fasting glucose and free fatty acid [12]. Collectively, the association between hsCRP and OCN was not clearly studied in adult males, especially those with systemic assessment of metabolic factors.

Currently, the putative receptor of OCN (GPRC6A) has been found to be expressed in leukocytes and active in cell mediated immunity, while it is not clear whether OCN or its receptors is active in humoral immunity. The complement system, as a part of the innate immunity, has been found to be involved in the development of metabolic complications [13]. Circulating C3 strongly predicts the cluster of MetS, independent of CRP [14]. Elevated serum C3 confers risk of coronary heart disease, independent of CRP as well [15]. The potential association of OCN with $\mathrm{C} 3$ may be distinctive from its association with CRP. Moreover, the adaptive immunity is also involved in the development of MetS. Among the immunoglobulins produced by B cells, IgM, as the first antibody produced during an immune response after an initial antigen counter, is associated with MetS and its individual components in males [16]. IgA concentrations above the reference range are common among male diabetics and associated with diabetic complications [17]. IgG, as the most abundant antibody in the circulation, is also involved in the development of hyperglycemia and obesity [18]. Transfer of IgG from diet-induced obese (DIO) mice rapidly induces insulin resistance and glucose intolerance [19], which could be improved by administration of exogenous OCN $[1,20]$. Nevertheless, human studies of association between OCN and inflammatory factors are still lacking. The evidence was so limited to support the hypothesis of potential involvement of OCN in the humoral immunity. So we conducted the present study to explore the association between OCN, hsCRP, complements (C3 and C4), IgM, IgG, IgA, and IgE in adult males. To the best of our knowledge, we are the first to explore the association between OCN and inflammatory factors with systemic assessment of metabolic factors. Our study would provide potential clues of cross talk between bone and immune system and partly support the increasing knowledge in osteoimmunology [21].

\section{Materials and Methods}

2.1. Study Population. Our analyses were based on the Fangchenggang Area Male Health and Examination Survey, a population-based cohort study in southern area of Guangxi, China [22]. It was a comprehensive demographic and health survey designed to investigate the effects of environmental and genetic factors and their interaction with the development of age-related chronic diseases. Briefly, a consecutive series of 4,303 men aged 17-88 years participated in the routine physical examination at the Medical Centre in Fangchenggang First People's Hospital from September 2009 to December 2009. A total of 3,593 people completed the data collection with a response rate of $83.5 \%$ [23]. There were no significant differences between these people and those who did not complete the interviews. All the participants provided written informed consent, and the present study was approved by the ethics committee in Guangxi Medical University.
Participants with the following conditions were excluded in the present study: (1) currently diagnosed with myocardial infarction, congestive heart failure, stroke, hyperparathyroidism, hyperthyroidism, rheumatoid arthritis, acquired immune deficiency syndrome, and cancer, or in current infection such as influenza, bronchitis, pneumonia, and urethritis; (2) with history of allergic diseases such as asthma and allergic rhinitis, or history of osteoporosis and bone fractures, or taking medications known to influence bone metabolism, such as vitamin $\mathrm{D}$, calcium, calcitonin, bisphosphonate, androgen, estrogen, or corticosteroids; (3) in current treatment with herbal remedies, or with medication including psychotropic drugs, opioids, cimetidine, spironolactone, antibiotics, and nonsteroidal anti-inflammatory drugs; (4) with impaired hepatic function (alanine transaminase $>2.0$ times upper limit of normal) or with impaired renal function (serum creatinine $>178 \mu \mathrm{mol} / \mathrm{L}$ ); and (5) having drunk beer, wine, or hard liquor the day before serologic examination. Finally, a total of 2,043 men aged 20-69 years with complete data were available for the present analysis.

2.2. Data Collection. A complete physical examination was performed on each subject. The standardized face-to-face questionnaire was used by trained physicians to collect information including smoking status, alcoholic drinking behavior, and health status. Current smokers were defined as smoking at least once a day and lasting for more than 6 months, while former smokers were those who have ceased smoking at least 6 months [24]. Alcoholic drinkers were defined as those who had ever consumed 3 or more drinks (beer, wine, and hard liquor) weekly and done so for 6 consecutive months $[24,25]$. The details of anthropometric measurements, including height, weight, (WC), and blood pressure (BP), were carried out by trained physicians. Body mass index (BMI) was calculated as weight in kilograms divided by the square of height in meters. WC was measured at the midpoint between the lower rib margin and the iliac. Both systolic blood pressure (SBP) and diastolic blood pressure (DBP) were measured twice after resting for more than 15 minutes and the average values were obtained.

2.3. Serum Assay. The description of the laboratory test has been previously reported in detail [5]. Briefly, about $10 \mathrm{~mL}$ overnight venous blood specimens were obtained between $8 \mathrm{AM}$ and $10 \mathrm{AM}$ and transported frozen to the testing center of Department of Clinical Laboratory at the First Affiliated Hospital of Guangxi Medical University in Nanning in two hours. Serums were centrifuged within 15-25 min and stored at $-80^{\circ} \mathrm{C}$ until analysis. High density lipoprotein cholesterol (HDL-c), triglycerides, and glucose were measured enzymatically on a Dimension-RxL Chemistry Analyzer (Dade Behring, Newark, Delaware) in the Department of Clinical Laboratory at the Fangchenggang First People's Hospital. Serum OCN and insulin were measured with electrochemiluminescence immunoassay on COBAS 6000 system E601 (Elecsys module) immunoassay analyzer (Roche Diagnostics, GmbH, Mannheim, Germany) with the same batch of reagents, and the interassay coefficient of variation was $4.5 \%$ and $3.2 \%$, respectively. The hsCRP, C3, C4, $\lg$, $\lg$, $\lg \mathrm{A}$, 
and IgE levels were detected using the immunoturbidimetric assay on the Hitachi 7600 autoanalyzer (Hitachi Corp, Tokyo, Japan), and the interassay coefficient of variation was $2.20 \%$, $2.16 \%, 2.51 \%, 4.67 \%, 4.97 \%, 3.57 \%$, and $3.30 \%$, respectively. HOMA-IR of insulin resistance was calculated as follows: ([insulin] $*$ [glucose]/22.5) [26].

2.4. Statistical Methods. All statistical analyses were performed using SPSS version 16.0 software (SPSS Inc., Chicago, Illinois). The distribution of continuous variables was examined by Shapiro-Wilks test. Variables were described as proportions for categorical variables and as medians and interquartile ranges for continuous variables with skewed distribution. Because the distribution of OCN levels was skewed, it was transformed by the natural logarithm. In order to maintain the symmetry and comparability of per-uniteffect estimates, the inflammatory factors including hsCRP, complements (C3, C4), and immunoglobulins (IgM, IgG, $\operatorname{Ig} \mathrm{A}$, and $\operatorname{IgE}$ ) were all $\log$-transformed in the following analysis. At first, correlations of metabolic factors with OCN and inflammatory factors were examined, and the ageadjusted Pearson's coefficients $(r)$ were presented. Then, the associations between OCN and inflammatory factors were evaluated in three linear regression models, and the beta coefficient $(\beta)$ and 95\% CI were present. Briefly, the first model was adjusting for age, then further adjusting for $\mathrm{BMI}$, and further adjusting for other confounders including triglycerides, HDL, fasting glucose, insulin, smoking status, SBP, DBP, and alcoholic drinking behavior. In the subgroup analysis, the associations between OCN and hsCRP, IgE were repeated in the final model adjusting for age, BMI, insulin, smoking status, and alcoholic drinking behavior. As shown in our previous study [22], MetS was defined based on the updated report of National Cholesterol Education Program Adult Treatment Panel III for Asian Americans [27], as having 3 or more of the following components: (1) central obesity: $\mathrm{WC} \geq 90 \mathrm{~cm}$, (2) hypertriglyceridemia: triglycerides $\geq 1.7 \mathrm{mmol} / \mathrm{L}$, (3) low HDL: HDL < $1.03 \mathrm{mmol} / \mathrm{L}$, (4) elevated BP: SBP/DBP $\geq 130 / 85 \mathrm{mmHg}$ or current use of antihypertensive medications, and (5) hyperglycemia: fasting glucose at $\geq 5.6 \mathrm{mmol} / \mathrm{L}$ or being previously diagnosed with type 2 diabetes mellitus or on oral antidiabetic agents or insulin. Participants were defined as having normal glucose tolerance (NGT, fasting glucose $<5.6 \mathrm{mmol} / \mathrm{L}$ ) or impaired fasting glucose (IFG, fasting glucose $=5.6-7.0 \mathrm{mmol} / \mathrm{L}$ ) or unknown diabetes (fasting glucose $>7.0 \mathrm{mmol} / \mathrm{L}$ ) according to American Diabetes Association [28]. Participants were also classified as obese on the basis of the Asian definition of $\mathrm{BMI} \geq 27.5 \mathrm{~kg} / \mathrm{m}^{2}$ [29]. Statistical tests were two-sided, and a $P<0.05$ was considered statistically significant.

\section{Results}

The baseline demographic characteristic of the 2,043 participants was shown in Table 1. Overall, the median age of the sample was 36 years (interquartile range: 29-45), and median serum OCN was $22.9 \mathrm{ng} / \mathrm{mL}$ (interquartile range: 18.8-28.6). OCN was significantly correlated with age $(r=-0.341, P<$ $0.001)$. Table 2 provided the age-adjusted Spearman partial
TABLE 1: Baseline demographic characteristics $(n=2,043)$.

\begin{tabular}{|c|c|}
\hline Variable & $\begin{array}{c}\text { Median (25-75th } \\
\text { percentiles) or } n \\
(\%)\end{array}$ \\
\hline Age (yr) & $36(29-45)$ \\
\hline$<30$ & $554(27.1)$ \\
\hline $30-40$ & $773(37.8)$ \\
\hline$>40$ & $716(35.0)$ \\
\hline \multicolumn{2}{|l|}{ Smoking status } \\
\hline Current smoker & $1028(50.3)$ \\
\hline Former smoker & $84(4.1)$ \\
\hline Never smoked & $931(45.6)$ \\
\hline \multicolumn{2}{|l|}{ Alcoholic drinker } \\
\hline Yes & $1750(85.7)$ \\
\hline No & $29(14.3)$ \\
\hline \multicolumn{2}{|l|}{ Physical examination } \\
\hline Body mass index $\left(\mathrm{kg} / \mathrm{m}^{2}\right)$ & $23.1(20.8-25.5)$ \\
\hline Waist circumference $(\mathrm{cm})$ & $80.5(73.5-87.5)$ \\
\hline Systolic blood pressure (mmHg) & $120(110-126)$ \\
\hline Diastolic blood pressure ( $\mathrm{mmHg}$ ) & $80(70-80)$ \\
\hline Osteocalcin (ng/mL) & $22.9(18.8-28.6)$ \\
\hline \multicolumn{2}{|l|}{ Inflammatory factors } \\
\hline High sensitivity C-reactive protein (mg/L) & $0.55(0.25-1.28)$ \\
\hline Complement C3 (IU/mL) & $1.11(0.97-1.26)$ \\
\hline Complement C4 (IU/mL) & $0.32(0.27-0.38)$ \\
\hline $\operatorname{IgM}(\mathrm{IU} / \mathrm{mL})$ & $1.23(0.9-1.7)$ \\
\hline $\operatorname{IgG}(\mathrm{IU} / \mathrm{mL})$ & $13.2(11.5-14.9)$ \\
\hline $\operatorname{IgA}(\mathrm{IU} / \mathrm{mL})$ & $2.36(1.79-2.97)$ \\
\hline $\operatorname{IgE}(\mathrm{IU} / \mathrm{mL})$ & $126.6(51.8-317.0)$ \\
\hline \multicolumn{2}{|l|}{ Metabolic factors } \\
\hline $\mathrm{HDL}(\mathrm{mmol} / \mathrm{L})$ & $1.35(1.18-1.57)$ \\
\hline Triglycerides (mmol/L) & $1.13(0.78-1.76)$ \\
\hline Fasting glucose $(\mathrm{mmol} / \mathrm{L})$ & $5.2(4.9-5.6)$ \\
\hline Insulin (mlU/L) & $6.5(4.42-9.76)$ \\
\hline HOMA & $0.83(0.57-1.27)$ \\
\hline
\end{tabular}

correlation coefficients between OCN, hsCRP, complements (C3, C4), IgM, IgG, IgA, IgE, and metabolic factors. The OCN was inversely correlated with hsCRP $(r=-0.144, P<0.001)$, C3 $(r=-0.123, P<0.001)$, C4 $(r=-0.073, P=0.001)$, and $\operatorname{IgE}(r=-0.057, P=0.034)$, while being positively correlated with $\operatorname{IgG}(r=0.048, P=0.034)$. In terms of metabolic factors, OCN was inversely correlated with BMI, WC, SBP, DBP, glucose, triglyceride, HOMA-IR, and insulin, while these metabolic factors were positively correlated with hsCRP, C3, and C4. Moreover, IgG was inversely correlated with BMI, and IgM was inversely correlated with WC, while IgE was inversely correlated with BMI and WC.

The associations between OCN and hsCRP, immunoglobulins, and complements were analyzed in the linear regression models as shown in Table 3. OCn was significantly associated with age $(\beta=-0.010,95 \% \mathrm{CI}=-0.011,-0.009$, 
TABLE 2: Age-adjusted Spearman partial correlations between log (osteocalcin), log (hsCRP), $\log$ (inflammatory factors), and log (metabolic factors).

\begin{tabular}{|c|c|c|c|c|c|c|c|c|}
\hline & Osteocalcin & hsCRP & $\mathrm{C} 3$ & $\mathrm{C} 4$ & $\operatorname{Ig} M$ & $\operatorname{IgG}$ & $\operatorname{Ig} \mathrm{A}$ & $\operatorname{Ig} \mathrm{E}$ \\
\hline Osteocalcin & & $-0.144^{\mathrm{c}}$ & $-0.123^{c}$ & $-0.073^{b}$ & 0.007 & $0.048^{\mathrm{a}}$ & 0.014 & $-0.057^{\mathrm{a}}$ \\
\hline BMI & $-0.292^{c}$ & $0.312^{c}$ & $0.402^{\mathrm{c}}$ & $0.223^{c}$ & -0.037 & $-0.050^{\mathrm{a}}$ & -0.022 & $-0.047^{\mathrm{a}}$ \\
\hline WC & $-0.267^{\mathrm{c}}$ & $0.320^{c}$ & $0.407^{\mathrm{c}}$ & $0.227^{\mathrm{c}}$ & $-0.061^{\mathrm{b}}$ & -0.092 & -0.011 & $-0.056^{\mathrm{b}}$ \\
\hline SBP & $-0.059^{\mathrm{b}}$ & $0.109^{c}$ & $0.127^{c}$ & $0.083^{c}$ & -0.035 & -0.023 & 0.042 & 0.007 \\
\hline DBP & $-0.113^{\mathrm{c}}$ & $0.129^{c}$ & $0.169^{c}$ & $0.085^{c}$ & $-0.056^{\mathrm{a}}$ & -0.014 & $0.056^{\mathrm{a}}$ & -0.013 \\
\hline Glucose & $-0.107^{\mathrm{c}}$ & $0.107^{c}$ & $0.121^{\mathrm{c}}$ & $0.062^{\mathrm{b}}$ & -0.001 & 0.001 & $0.057^{\mathrm{a}}$ & 0.028 \\
\hline Triglyceride & $-0.199^{c}$ & $0.223^{c}$ & $0.279^{c}$ & $0.110^{c}$ & -0.006 & -0.130 & -0.039 & -0.022 \\
\hline HDL & $0.079^{c}$ & $-0.180^{c}$ & $-0.246^{\mathrm{c}}$ & $-0.152^{c}$ & -0.014 & -0.016 & -0.036 & $0.052^{\mathrm{a}}$ \\
\hline HOMA & $-0.145^{\mathrm{c}}$ & $0.213^{c}$ & $0.278^{\mathrm{c}}$ & $0.138^{c}$ & -0.011 & -0.025 & $0.061^{\mathrm{b}}$ & -0.007 \\
\hline Insulin & $-0.140^{\mathrm{c}}$ & $0.247^{\mathrm{c}}$ & $0.358^{c}$ & $0.152^{c}$ & $-0.057^{\mathrm{a}}$ & -0.029 & $0.045^{\mathrm{a}}$ & -0.009 \\
\hline
\end{tabular}

WC: waist circumference, SDP: systolic blood pressure, and DBP: diastolic blood pressure.

${ }^{\mathrm{a}} P<0.05,{ }^{\mathrm{b}} P<0.01$, and ${ }^{\mathrm{c}} P<0.001$, respectively (all two-tailed).

TABLE 3: Linear regression coefficients and 95\% CI for log (osteocalcin) as the dependent variable and log (hsCRP) and log (inflammatory factors) as independent variables.

\begin{tabular}{|c|c|c|c|c|c|c|}
\hline \multirow{2}{*}{ Inflammatory factors } & \multicolumn{2}{|c|}{ Model 1} & \multicolumn{2}{|c|}{ Model 2} & \multicolumn{2}{|c|}{ Model 3} \\
\hline & $\beta$ & $95 \% \mathrm{CI}$ & $\beta$ & $95 \% \mathrm{CI}$ & $\beta$ & $95 \% \mathrm{CI}$ \\
\hline hsCRP & $-0.034^{\mathrm{c}}$ & $(-0.044,-0.024)$ & $-0.014^{\mathrm{a}}$ & $(-0.024,-0.003)$ & $-0.012^{\mathrm{a}}$ & $(-0.023,-0.002)$ \\
\hline $\mathrm{C} 3$ & $-0.171^{\mathrm{c}}$ & $(-0.231,-0.111)$ & -0.010 & $(-0.013,-0.010)$ & -0.017 & $(-0.082,0.048)$ \\
\hline $\mathrm{C} 4$ & $-0.079^{\mathrm{b}}$ & $(-0.126,-0.033)$ & -0.009 & $(-0.055,0.037)$ & -0.014 & $(-0.061,0.032)$ \\
\hline $\operatorname{IgM}$ & 0.004 & $(-0.022,0.031)$ & -0.002 & $(-0.028,0.023)$ & -0.006 & $(-0.031,0.020)$ \\
\hline IgG & $0.068^{\mathrm{a}}$ & $(0.006,0.129)$ & 0.047 & $(-0.013,-0.010)$ & 0.026 & $(-0.035,0.087)$ \\
\hline $\operatorname{IgA}$ & 0.012 & $(-0.024,0.047)$ & 0.006 & $(-0.027,-0.040)$ & 0.000 & $(-0.034,0.035)$ \\
\hline IgE & $-0.013^{\mathrm{b}}$ & $(-0.022,-0.003)$ & $-0.016^{\mathrm{b}}$ & $(-0.025,-0.007)$ & $-0.015^{\mathrm{a}}$ & $(-0.024,-0.006)$ \\
\hline
\end{tabular}

Model 1 was adjusted for age; model 2 was further adjusted for BMI; model 3 was adjusted for model 2 plus triglycerides, HDL, fasting glucose, insulin, smoking status, systolic blood pressure, diastolic blood pressure, and alcoholic drinking behavior.

${ }^{\mathrm{a}} P<0.05,{ }^{\mathrm{b}} P<0.01$, and ${ }^{\mathrm{c}} P<0.001$, respectively (all two-tailed).

and $P<0.001)$. In age-adjusted model, OCN was inversely associated with hsCRP $(\beta=-0.034,95 \% \mathrm{CI}=-0.044$ to -0.024 , and $P<0.001), \mathrm{C} 3(\beta=-0.171,95 \% \mathrm{CI}=-0.231$ to -0.111 , and $P<0.001), \mathrm{C} 4(\beta=-0.079,95 \% \mathrm{CI}=$ -0.126 to -0.033 , and $P<0.001)$, and $\operatorname{IgE}(\beta=-0.013$, $95 \% \mathrm{CI}=-0.022,-0.003$, and $P=0.005)$. In the ageBMI-adjusted model, the inverse association between OCN and hsCRP $(\beta=-0.014,95 \% \mathrm{CI}=-0.024,-0.003$, and $P<0.001)$ and $\operatorname{IgE}(\beta=-0.016,95 \% \mathrm{CI}=-0.025,-0.007$, and $P=0.001$ ) remained statistically significant, and these associations were not attenuated in the model with further adjusting for triglycerides, HDL, glucose, insulin, SBP, DBP, smoking status, and alcoholic drinking behavior.

The associations between OCN and hsCRP and IgE were further explored in the subgroups as shown in Table 4. The inverse association between OCN and hsCRP was obviously observed in men with MetS $(\beta=-0.046,95 \% \mathrm{CI}=-0.088$ to -0.004 , and $P=0.032)$ and men with hyperglycemia $(\beta=$ $-0.039,95 \% \mathrm{CI}=-0.059$ to -0.018 , and $P<0.001)$ or IFG $(\beta=-0.035,95 \% \mathrm{CI}=-0.057$ to -0.0148 , and $P=0.001)$ or $\mathrm{BMI} \geq 27.5 \mathrm{~kg} / \mathrm{m}^{2}(\beta=-0.039,95 \% \mathrm{CI}=-0.075$ to -0.002 , and $P=0.039)$. Meanwhile, the inverse association between OCN and IgE was only observed in men without MetS $(\beta=$ $-0.017,95 \% \mathrm{CI}=-0.027$ to -0.008 , and $P<0.001)$ and men with NGT $(\beta=-0.019,95 \% \mathrm{CI}=-0.029$ to -0.008 , and $P<$ $0.001)$ or $\mathrm{BMI}<27.5 \mathrm{~kg} / \mathrm{m}^{2}(\beta=-0.015,95 \% \mathrm{CI}=-0.025$ to -0.006 , and $P=0.020$ ) or men without each individual component of MetS factors. Moreover, the inverse association of OCN with both hsCRP and IgE was statistically significant in men with only one component of MetS factors.

\section{Discussions}

In this large cross-sectional study of Chinese male population with systemic assessment of metabolic factors, serum OCN was inversely associated with hsCRP, especially in men with IFG, hyperglycemia, or MetS. The potential association between OCN and C3 may be mediated by obesity. OCN was not associated with IgM and IgA but inversely associated with $\operatorname{IgE}$ in men with a normal metabolic profile.

Although the inverse association between OCN and hsCRP has been reported, we are the first to show this association in adult males with prediabetes or MetS. As shown in another Chinese study enrolling adult males with risk factors for cardiovascular diseases, the age-adjusted inverse association between OCN and CRP was observed in men with NGT and normal BMI but was not statistically significant after further adjusting for visceral fat area [12]. In the present 
TABLE 4: Multivariant-adjusted association between $\log$ (osteocalcin) and $\log (\mathrm{hsCRP})$ and $\log (\operatorname{IgE})$ in subgroups.

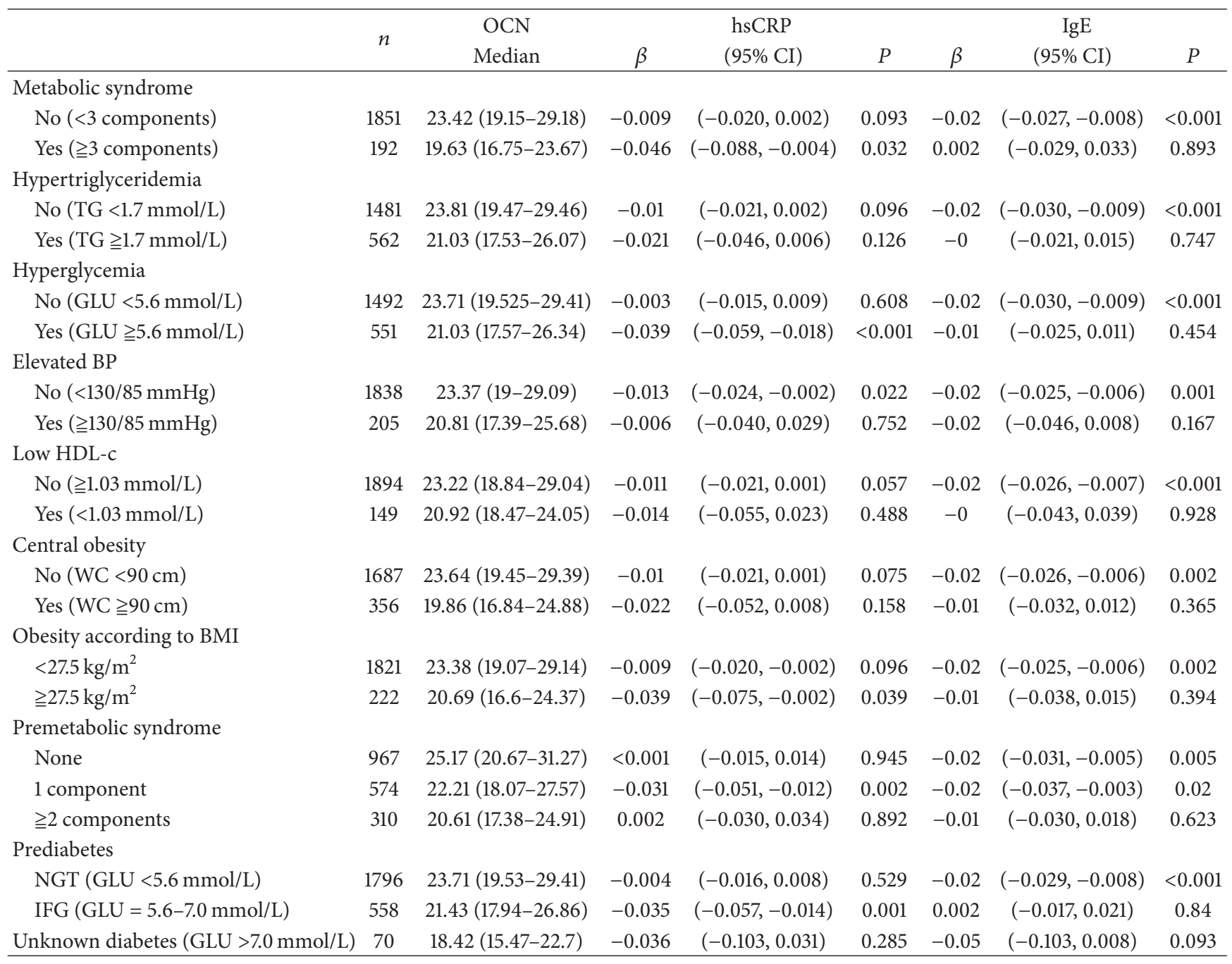

Adjusted for age, BMI, insulin, smoking status, and alcoholic drinking behavior.

OCN: osteocalcin, hsCRP: high sensitive C-reaction protein, TG: triglycerides, GLU: fasting glucose, BP: blood pressure, HDL-c: high density lipoprotein cholesterol, WC: waist circumference, BMI: body mass index, NGT: normal glucose tolerance, and IFG: impaired fasting glucose.

study, the inverse association between OCN and hsCRP was statistically significant in men with IFG, hyperglycemia, or MetS, even after adjusting metabolic confounders including BMI or WC. In the European study of nondiabetic overweight and obese adults, OCN was inversely associated with hsCRP after adjusting for age, physical activity, and fat mass, but this significant association was only observed in the female subjects [9], consistent with the results from postmenopausal women [8]. On the one hand, the sample size of male subjects $(N=116)$ in this study was probably too small to detect the genuine association between OCN and CRP in males. On the other hand, gender difference may exist in the potential association between OCN and CRP, as postmenopausal women had a lower level of $\mathrm{CON}$ and a higher level of CRP than middle-aged and elderly men [8], while young women had a higher level of OCN and a lower level of CRP than young men [9]. Similarly, ethnic/racial difference may exist as well $[11,30]$. As shown in a multiethnic study of school children, OCN was significantly higher in East Asian Americans than the other ethnic/racial groups [30]. Although the gender or ethnic/racial difference has not been confirmed, the inverse association between OCN and hsCRP in IFG or hyperglycemia may shed new light on the anti-inflammatory role of OCN in maintaining glucose homeostasis.

To the best of our knowledge, we are the first to explore the association between OCN and complements $\mathrm{C} 3, \mathrm{C} 4$. Consistent with previous studies, we found that C3 was positively associated with metabolic factors including fasting triglycerides, WC, HOMA-IR, and BMI [14]. So was the association between $\mathrm{OCN}$ and MetS, as we previously showed that low level of serum OCN was a risk factor for MetS [5]. Thus, it was not surprising to find out that OCN was inversely associated with C3 in the present study. However, this age-adjusted association was not statistically significant after further adjusting for BMI or WC. Because adipose tissue can produce both $\mathrm{C} 3$ and OCN, this association between 
OCN and C3 may be affected by BMI or WC, two important proxies of obesity. Considering the contribution of complement in the low-grade inflammation associated with obesity, OCN may be involved in the pathways of complement activation during metabolic inflammation [31]. Although we cannot rule out the possibility that OCN was associated with C3 independent of metabolic factors, OCN as one of the biomarkers secreted by osteoblast cells may be related to the inflammatory process involving complements [32].

Immunoglobulins were potentially associated with MetS, but their association with OCN has not been studied before. As shown in the previous study, serum IgM concentration was positively associated with MetS in an adult population [16]. In the mice model, the hyperglycemia alone can delay production of IgM [33]. Although OCN may play a beneficial role in the development of hyperglycemia, its effect on the production of IgM has not been identified. Our findings suggested OCN was not associated with $\operatorname{IgM}$, but further researches are needed to verify it. In DIO mice, not only reduced production of IgM but also increased IgG secretion were observed [19]. The transfer of purified IgG from DIO mice but not from lean mice induces an Fcdependent worsening of insulin resistance, suggesting the linkage between IgG and obesity-related insulin resistance [19]. In the present study, the association between IgG and OCN was initially observed, but this age-adjusted association was not statistically significant after further adjusting for BMI or WC. Adipose tissue seems to play a crucial role in the association between IgG and OCN [18]. Because there are $\mathrm{Fc}$ receptors in human adipose tissue [34], the IgG may have direct effects on adipocyte function and leads to OCN production [35]. Because IgA exists primarily as a monomer in serum, its exposed Fc domains may relate to the Fcdependent function on adipocytes as well. But in the present study, IgA was not associated with OCN, probably due to the $\mathrm{J}$ chain in the polymeric forms of IgA, which bonds with the Fc domains of IgA and disturbs the Fc-dependent function on adipocytes. In the present study, only IgE was significantly associated with OCN, and this association was obviously observed in healthy adults without each component of MetS. MetS is characterized by an aggregate of interrelated metabolic factors consisting of insulin resistance, visceral obesity, and atherogenic dyslipidemia. The significant association between OCN and $\mathrm{IgE}$ was still observed in men with only one component of MetS. These findings may suggest the important role of OCN and IgE in the development of MetS. As shown in the rat model, the cholesterol accumulation within the macrophages of atherosclerotic lesion was IgEdependent [36]. Although the effect of OCN on the IgEactivated mast cells has not been identified, effective mast cell inhibitor medications used in preformed obesity and diabetes in experimental models offer hope to patients with these common chronic inflammatory diseases [37].

Our study was strengthened by the large sample size of the cohort population with a wide range of age. We also controlled the potential confounding of traditional metabolic factors in data analyses. However, we recognized several limitations in the present study. Firstly, given the nature of crosssectional study, we could not establish causal relationships between OCN and the inflammatory factors. Secondly, we only measured hsCRP, complements, and immunoglobulins as inflammatory markers but did not evaluate other markers such as tumor necrosis factor $\alpha$ and IL-6, which were potentially associated with obesity or type 2 diabetes [38]. Particularly, assessment of testosterone would be of high value in systemic inflammation-based vasculopathy and metabolic syndrome [39]. Last but not least, the study population consisted solely of men, and only total OCN was measured but not its uncarboxylated or carboxylated form. Considering the potential differences between total OCN and uncarboxylated OCN and the differences between males and females, further well-designed study is needed to explore the role of $\mathrm{OCN}$ in the inflammation related to metabolic disorders. Despite these limitations, this is the first crosssectional study to investigate the relation between OCN and inflammatory factors in general population.

In conclusion, our study tried to clarify the role of metabolic factors in the association between OCN and the inflammatory factors. We find out that serum OCN was not associated with $\operatorname{IgM}$ and $\operatorname{IgA}$, while its potential association with IgG and C3 was probably mediated by obesity. Moreover, serum OCN was inversely associated with hsCRP in men with IFG, hyperglycemia, or MetS, while being inversely associated with IgE in men with a normal metabolic profile. Our findings may support the possibility of using OCN in the prediction or treatment for metabolic disorders.

\section{Conflict of Interests}

The authors declare that they have no conflict of interests.

\section{Acknowledgments}

The authors express their sincere thanks to the local research teams from Fangchenggang First People's Hospital, Fangchenggang, China, for their contribution to the survey. Thanks are due to support from National Natural Science Foundation of China (81460159, 81260130, 81060234, 30945204, 30360124, and 30260110), Key Program and University Talents Highland Innovation Team of Guangxi (2012012D003, GJR201147-09), Chairman Science and Technology Fund and Tackle Program of Guangxi (1116-03, GKG1298003-07-01), Guangxi Provincial Department of Finance and Education (2009GJCJ150), Guangxi Natural Science Foundation (2014GXNSFBA118150, 2012GXNSFDA053016), Guangxi Science Fund for Distinguished Young Scholars (2012GXNSFFA060009), Guangxi 2011 Graduate Education Innovation Program (2011105981002M202), and Innovation Project of Guangxi Graduate Education (02304001012).

\section{References}

[1] N. K. Lee, H. Sowa, E. Hinoi et al., "Endocrine regulation of energy metabolism by the skeleton," Cell, vol. 130, no. 3, pp. 456469, 2007.

[2] G. Fantuzzi, “The sound of health," Frontiers in Immunology, vol. 5, article 351, 2014. 
[3] M. Pi and L. D. Quarles, "Novel bone endocrine networks integrating mineral and energy metabolism," Current Osteoporosis Reports, vol. 11, no. 4, pp. 391-399, 2013.

[4] M. Ferron and J. Lacombe, "Regulation of energy metabolism by the skeleton: osteocalcin and beyond," Archives of Biochemistry and Biophysics, vol. 561, pp. 137-146, 2014.

[5] A. Tan, Y. Gao, X. Yang et al., "Low serum osteocalcin level is a potential marker for metabolic syndrome: results from a Chinese male population survey," Metabolism: Clinical and Experimental, vol. 60, no. 8, pp. 1186-1192, 2011.

[6] M. Pravenec, T. Kajiya, V. Zídek et al., "Effects of human Creactive protein on pathogenesis of features of the metabolic syndrome," Hypertension, vol. 57, no. 4, pp. 731-737, 2011.

[7] G. Schett, S. Kiechl, S. Weger et al., "High-sensitivity C-reactive protein and risk of nontraumatic fractures in the bruneck study," Archives of Internal Medicine, vol. 166, no. 22, pp. 2495-2501, 2006.

[8] L. Chen, Q. Li, Z. Yang et al., "Osteocalcin, glucose metabolism, lipid profile and chronic low-grade inflammation in middleaged and elderly Chinese," Diabetic Medicine, vol. 30, no. 3, pp. 309-317, 2013.

[9] A. J. Lucey, G. K. Paschos, I. Thorsdottir, J. A. Martínéz, K. D. Cashman, and M. Kiely, "Young overweight and obese women with lower circulating osteocalcin concentrations exhibit higher insulin resistance and concentrations of C-reactive protein," Nutrition Research, vol. 33, no. 1, pp. 67-75, 2013.

[10] P. D. Sarkar and A. B. Choudhury, "Relationships between serum osteocalcin levels versus blood glucose, insulin resistance and markers of systemic inflammation in central Indian type 2 diabetic patients," European Review for Medical and Pharmacological Sciences, vol. 17, no. 12, pp. 1631-1635, 2013.

[11] A. G. Pittas, S. S. Harris, M. Eliades, P. Stark, and B. DawsonHughes, "Association between serum osteocalcin and markers of metabolic phenotype," Journal of Clinical Endocrinology and Metabolism, vol. 94, no. 3, pp. 827-832, 2009.

[12] Y. Bao, X. Ma, R. Yang et al., "Inverse relationship between serum osteocalcin levels and visceral fat area in Chinese men," The Journal of Clinical Endocrinology \& Metabolism, vol. 98, no. 1, pp. 345-351, 2013.

[13] E. Hertle, C. D. A. Stehouwer, and M. M. J. van Greevenbroek, "The complement system in human cardiometabolic disease," Molecular Immunology, vol. 61, no. 2, pp. 135-148, 2014.

[14] A. Onat, G. Hergenç, G. Can, Z. Kaya, and H. Yüksel, "Serum complement C3: a determinant of cardiometabolic risk, additive to the metabolic syndrome, in middle-aged population," Metabolism: Clinical and Experimental, vol. 59, no. 5, pp. 628634, 2010.

[15] R. Ajjan, P. J. Grant, T. S. Futers et al., "Complement C3 and C-reactive protein levels in patients with stable coronary artery disease," Thrombosis and Haemostasis, vol. 94, no. 5, pp. 10481053, 2005.

[16] K. Song, H. Du, Q. Zhang et al., "Serum immunoglobulin $\mathrm{M}$ concentration is positively related to metabolic syndrome in an adult population. Tianjin Chronic Low-Grade Systemic Inflammation and Health (TCLSIH) cohort study," PLoS ONE, vol. 9, no. 2, Article ID e88701, 2014.

[17] S. Rodriguez-Segade, M. F. Camiña, A. Carnero et al., "High serum IgA concentrations in patients with diabetes mellitus: agewise distribution and relation to chronic complications," Clinical Chemistry, vol. 42, no. 7, pp. 1064-1067, 1996.

[18] D. A. Winer, S. Winer, M. H. Y. Chng, L. Shen, and E. G. Engleman, "B Lymphocytes in obesity-related adipose tissue inflammation and insulin resistance," Cellular and Molecular Life Sciences, vol. 71, no. 6, pp. 1033-1043, 2014.

[19] D. A. Winer, S. Winer, L. Shen et al., "B cells promote insulin resistance through modulation of $\mathrm{T}$ cells and production of pathogenic IgG antibodies," Nature Medicine, vol. 17, no. 5, pp. 610-617, 2011.

[20] B. Zhou, H. Li, L. Xu, W. Zang, S. Wu, and H. Sun, "Osteocalcin reverses endoplasmic reticulum stress and improves impaired insulin sensitivity secondary to diet-induced obesity through nuclear factor- $\kappa$ b signaling pathway," Endocrinology, vol. 154, no. 3, pp. 1055-1068, 2013.

[21] H. Takayanagi, "Osteoimmunology in 2014: two-faced immunology-from osteogenesis to bone resorption," Nature Reviews Rheumatology, vol. 11, no. 2, pp. 74-76, 2015.

[22] M. Liao, X. Guo, X. Yu et al., "Role of metabolic factors in the association between osteocalcin and testosterone in Chinese men," The Journal of Clinical Endocrinology \& Metabolism, vol. 98, no. 8, pp. 3463-3469, 2013.

[23] C. Wu, H. Zhang, Y. Gao et al., "The association of smoking and erectile dysfunction: results from the fangchenggang area male health and examination survey (FAMHES)," Journal of Andrology, vol. 33, no. 1, pp. 59-65, 2012.

[24] A. Gonzalez-Quintela, R. Alende, F. Gude et al., "Serum levels of immunoglobulins (IgG, IgA, IgM) in a general adult population and their relationship with alcohol consumption, smoking and common metabolic abnormalities," Clinical and Experimental Immunology, vol. 151, no. 1, pp. 42-50, 2008.

[25] H. M. Perry III, M. Horowitz, S. Fleming et al., "The effects of season and alcohol intake on mineral metabolism in men," Alcoholism: Clinical and Experimental Research, vol. 23, no. 2, pp. 214-219, 1999.

[26] D. R. Matthews, J. P. Hosker, A. S. Rudenski, B. A. Naylor, D. F. Treacher, and R. C. Turner, "Homeostasis model assessment: insulin resistance and beta-cell function from fasting plasma glucose and insulin concentrations in man," Diabetologia, vol. 28, no. 7, pp. 412-419, 1985.

[27] S. M. Grundy, J. I. Cleeman, S. R. Daniels et al., "Diagnosis and management of the metabolic syndrome: an American Heart Association/National Heart, Lung, and Blood Institute scientific statement," Circulation, vol. 112, no. 17, pp. 2735-2752, 2005.

[28] American Diabetes Association, "Standards of medical care in diabetes-2013," Diabetes Care, vol. 36, supplement 1, pp. S11S66, 2013.

[29] K. Shiwaku, E. Anuurad, B. Enkhmaa, K. Kitajima, and Y. Yamane, "Appropriate BMI for Asian populations," The Lancet, vol. 363, no. 9414, p. 1077, 2004.

[30] C. Boucher-Berry, P. W. Speiser, D. E. Carey et al., "Vitamin D, osteocalcin, and risk for adiposity as comorbidities in middle school children," Journal of Bone and Mineral Research, vol. 27, no. 2, pp. 283-293, 2012.

[31] B. Nilsson, O. A. Hamad, H. Ahlström et al., "C3 and C4 are strongly related to adipose tissue variables and cardiovascular risk factors," European Journal of Clinical Investigation, vol. 44, no. 6, pp. 587-596, 2014.

[32] P. Schoengraf, J. D. Lambris, S. Recknagel et al., "Does complement play a role in bone development and regeneration?" Immunobiology, vol. 218, no. 1, pp. 1-9, 2013.

[33] B. S. Nikolajczyk, M. Jagannathan-Bogdan, H. Shin, and R. Gyurko, "State of the union between metabolism and the immune system in type 2 diabetes," Genes and Immunity, vol. 12, no. 4, pp. 239-250, 2011. 
[34] J. Palming, B. G. Gabrielsson, E. Jennische et al., "Plasma cells and Fc receptors in human adipose tissue-lipogenic and anti-inflammatory effects of immunoglobulins on adipocytes," Biochemical and Biophysical Research Communications, vol. 343, no. 1, pp. 43-48, 2006.

[35] C. Foresta, G. Strapazzon, L. De Toni et al., "Evidence for osteocalcin production by adipose tissue and its role in human metabolism," Journal of Clinical Endocrinology and Metabolism, vol. 95, no. 7, pp. 3502-3506, 2010.

[36] H. Ma and P. T. Kovanen, "IgE-dependent generation of foam cells: an immune mechanism involving degranulation of sensitized mast cells with resultant uptake of LDL by macrophages," Arteriosclerosis, Thrombosis, and Vascular Biology, vol. 15, no. 6, pp. 811-819, 1995.

[37] J. Zhang and G.-P. Shi, "Mast cells and metabolic syndrome," Biochimica et Biophysica Acta: Molecular Basis of Disease, vol. 1822, no. 1, pp. 14-20, 2012.

[38] X. Wang, W. Bao, J. Liu et al., "Inflammatory markers and risk of type 2 diabetes: a systematic review and meta-analysis," Diabetes Care, vol. 36, no. 1, pp. 166-175, 2013.

[39] L. Antonio, F. C. Wu, T. W. O'Neill et al., "Associations between sex steroids and the development of metabolic syndrome: a longitudinal study in European men," The Journal of Clinical Endocrinology \& Metabolism, vol. 100, no. 4, pp. 1396-1404, 2015. 


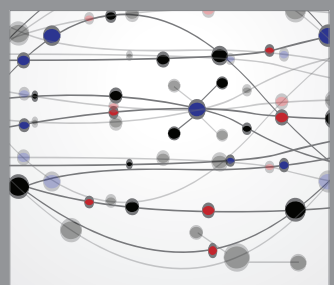

The Scientific World Journal
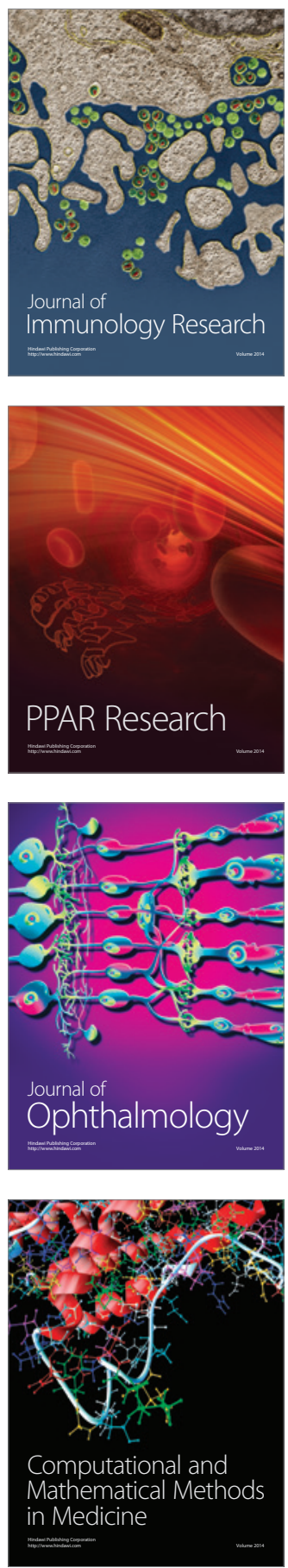

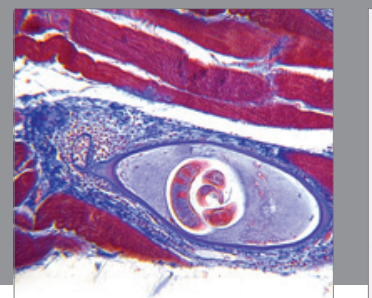

Gastroenterology

Research and Practice
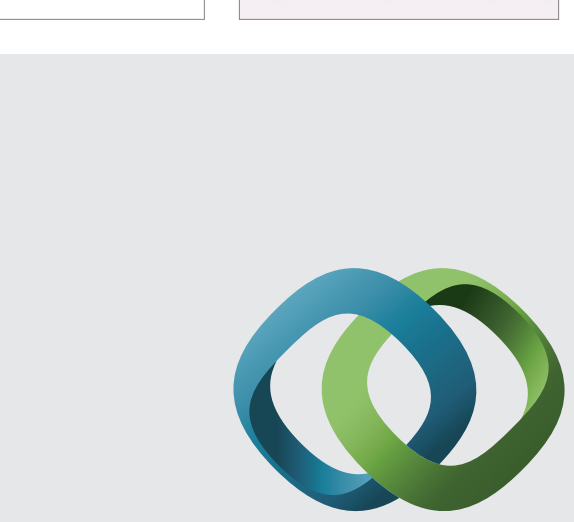

\section{Hindawi}

Submit your manuscripts at

http://www.hindawi.com
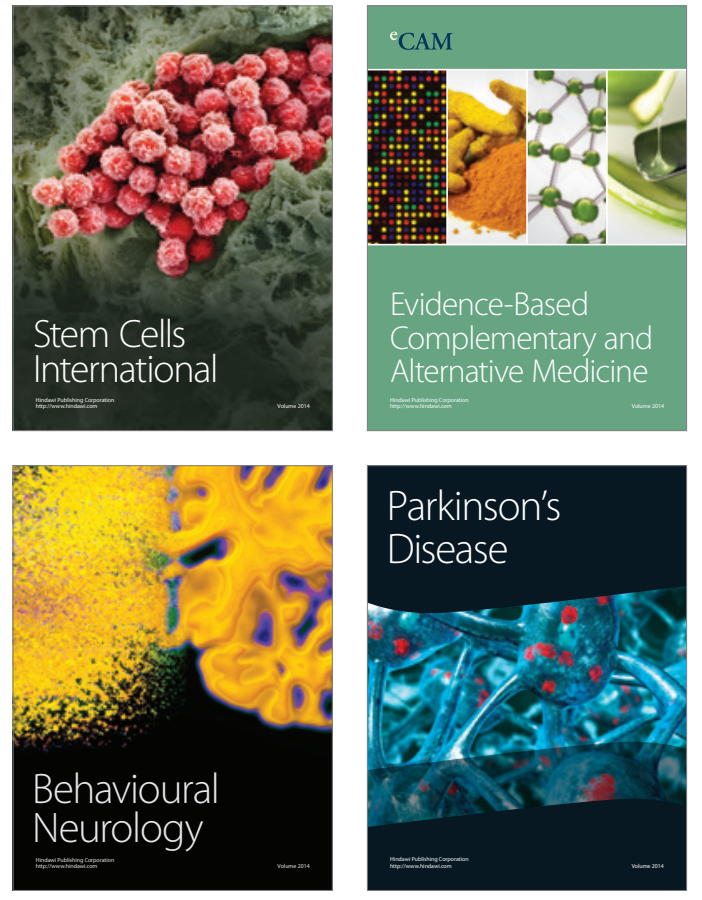
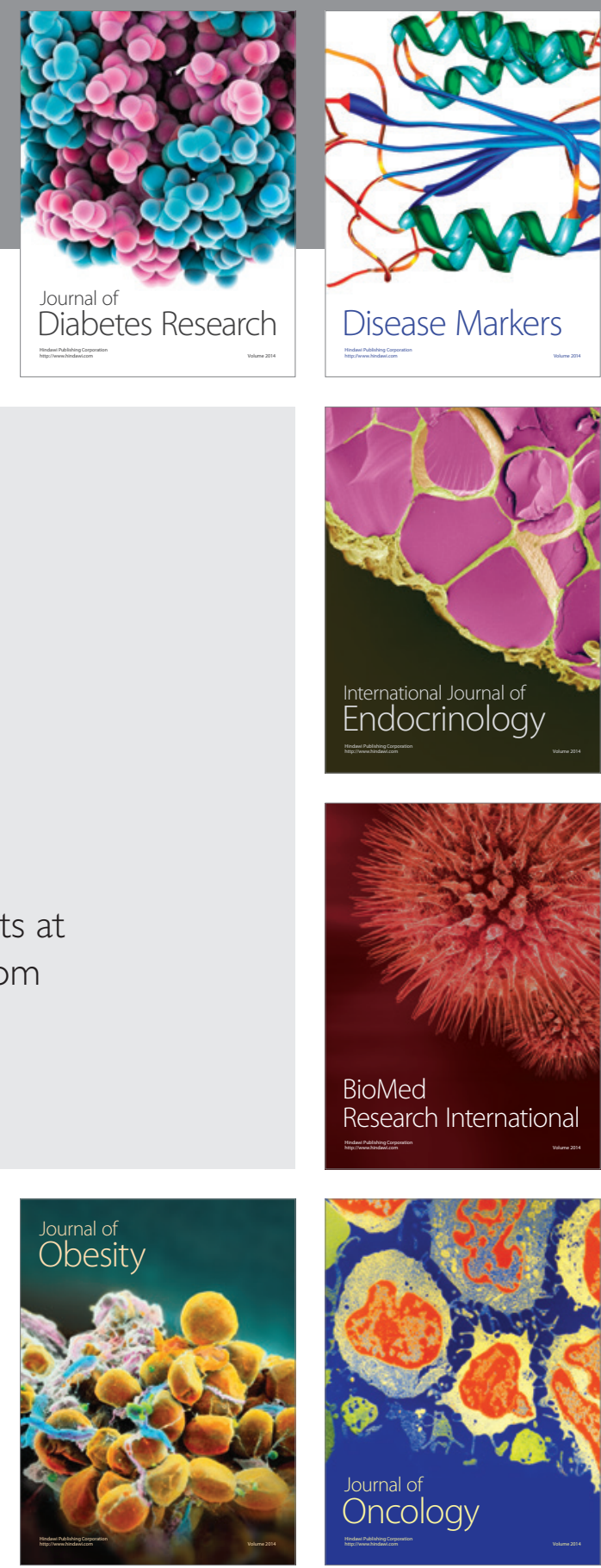

Disease Markers
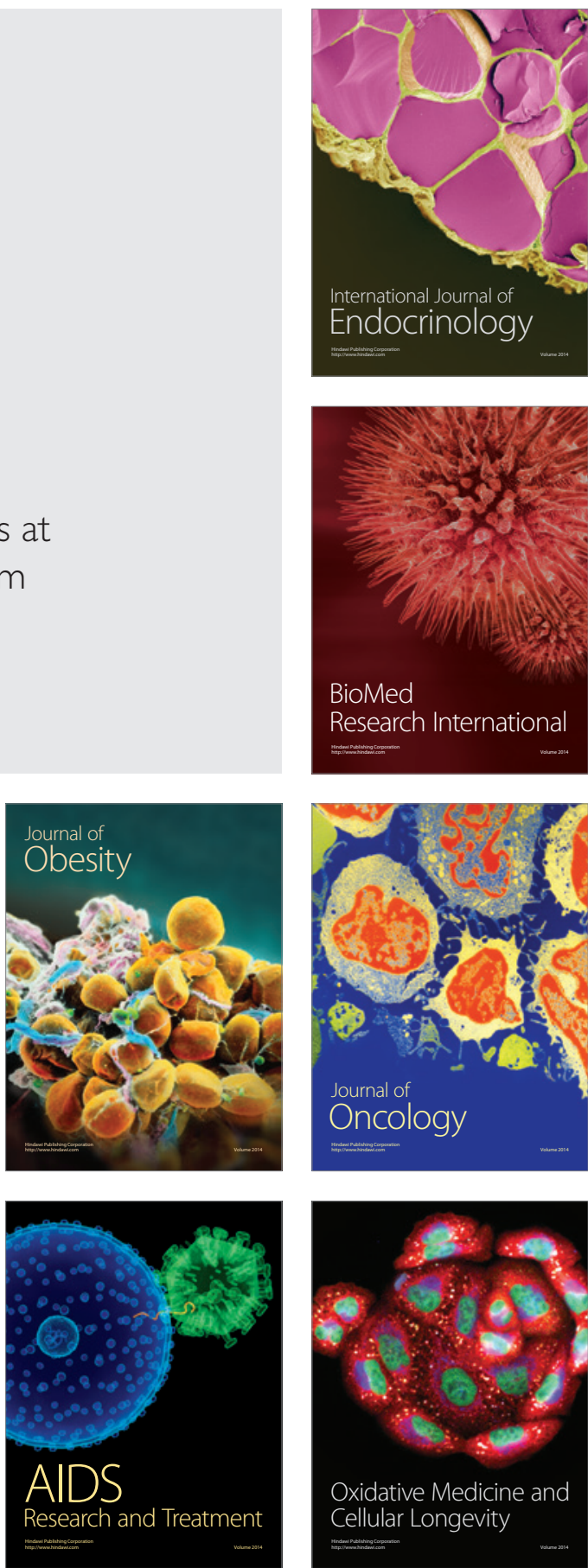